\title{
ASPECTOS PRODUTIVOS E COMERCIAIS DO PINHÃO NO ESTADO DO PARANÁ
}

\author{
Anadalvo Juazeiro dos Santos ${ }^{1}$ \\ Néder Maciel Corso ${ }^{2}$ \\ Gílson Martins \\ Eduardo Bittencourt ${ }^{4}$
}

\begin{abstract}
RESUMO
Este artigo tem por objetivo analisar aspectos produtivos e de comercialização do pinhão, semente da árvore conhecida vulgarmente como Pinheiro do Paraná (Araucária angustifolia (Bert.) O Kuntze). Esta espécie vegetal de porte arbóreo ocorre na região Sul e Sudeste do Brasil, principalmente nos Estados do Rio Grande do Sul, Santa Catarina e Paraná. Do ponto de vista metodológico foi primeiramente realizado um levantamento de dados econômicos sobre este produto nas instituições públicas do Estado do Paraná. A partir destes dados foram calculadas a Margem Bruta e o Markup de Comercialização. Também foi construído o fluxograma que apresenta os diversos elos que compõe a cadeia extrativa deste Produto Florestal Não Madeirável. Os resultados obtidos indicam que a maior parte da lucratividade no interior desta cadeia é apropriada pelos intermediários. Técnicas de conservação e industrialização devem ser desenvolvidas para promover a comercialização e uso do pinhão em outras épocas do ano, além da estação de produção. Isto tornaria o preço de mercado mais atraente incentivando a sua extração e comercialização.

Palavras-chave: Pinhão, Markup de comercialização, Margem de Comercialização, Pinheiro do Paraná
\end{abstract}

\section{PRODUCTIVE AND COMMERCIAL ASPECT OF PINHÃO IN PARANA STATE}

\begin{abstract}
This paper intends to give a marketing prime in the study of the pinhão, an edible seed from Parana-pine (Araucaria angustifolia (Bert.) O Kuntze).This species occurs in Southern Brazil, mainly along the States of Rio Grande do Sul, Santa Catarina and Parana.This study concentrated firstly in the agregation of data from publics institucions of Parana State. Secondly, it carries out the calculus of the index of gross commercialization margins and markup of commercialization. It also builds a flow concerning the various levels composing the marketing chain. Results obtained point out that the majority of rentability is apropriate for intermediary also the industralization techniques should be developed in order to promote the commercialization and use of pinhão in other periods of the year, besides the season of production. Addicionaly, there should be better prices for de producers to incentive the activity.
\end{abstract}

Key-words: Seeds, Margins, Markup, Parana - pine

\section{INTRODUÇÃO}

A Araucária (Araucaria angustifolia (Bert.) O.Kuntze) também conhecida como Pinheiro do Paraná é uma espécie geralmente encontrada em agrupamentos densos, principalmente na parte leste e central do planalto meridional do Brasil, abrangendo os estados do Paraná, Santa Catarina e Rio Grande do Sul, ocorrendo ainda, como manchas esparsas no sul do Estado de São Paulo e na Serra da Mantiqueira, estendendose até o sul de Minas Gerais e Rio de Janeiro. Entre as latitudes $25^{\circ} 30^{\prime}$ e $27^{\circ}$ sul, a araucária ocorre também na Província Argentina de Misiones. Existem também remanescentes no Paraguai na Bacia do Rio Paraná (Rizinni, 1971). No Brasil, a área de ocorrência natural da araucária era de aproximadamente 200.000 $\mathrm{km}^{2}$. Estima-se que em 1890, no Estado do

\footnotetext{
${ }^{1}$ Prof. Dr. do Curso de Pós-Graduação Eng.Florestal-UFPR ajsantos@ floresta.ufpr.br - Av. Lothário Meissner, 3400 -80210170, Curitiba Pr

${ }^{2}$ Eng Florestal Mestrando em Economia e Política Florestal - Engenharia Florestal - UFPR

${ }^{3}$ Engenheiro Florestal Mestrando em Economia e Política Florestal - Engenharia Florestal - UFPR

${ }^{4}$ Engenheiro Florestal - Engenharia Florestal - UFPR
} 
Paraná, a área de florestas naturais cobertas por esta espécie era de aproximadamente 73 $780 \mathrm{Km} 2$, tendo sido reduzida para $2594 \mathrm{~km}^{\circ}$ até o ano de 1995. (Sanquetta, 1999).

A floresta das araucárias é uma fonte de inúmeros sub-produtos florestais, tanto madeiráveis como não-madeiráveis. Dentre estes, destaca-se a erva-mate, que juntamente com a produção de madeira de araucária, cuja extração remonta o século XVI, tiveram um importante significado para o desenvolvimento econômico do estado.

O pinhão, semente da Araucaria angustifolia, é um alimento nutritivo bastante apreciado pela fauna silvestre e pelo homem. Apesar disto, talvez mais por falta de uma cultura de industrial do que por dificuldades técnicas em seu processamento, este não tem sido largamente empregado na culinária brasileira como o são as outras amêndoas. Seu consumo mais usual é na forma assada ou cozida, no entanto, algumas iguarias têm sido desenvolvidas com sua utilização. O consumo do pinhão faz parte da cultura da população, principalmente na região sul do país, sendo dessa forma imprescindível sua presença nas chamadas festas juninas. Dentre as festividades dedicadas ao pinhão vale destacar a tradicional festa do pinhão de Lages $-\mathrm{SC}$ e a feira do pinhão em Curitiba.

Apesar da sua importância históricocultural na alimentação da população dessas regiões, pouca atenção tem sido dada a pesquisas sobre os vários aspectos que envolvem seu consumo.

Neste contexto, a compreensão acerca do funcionamento desta cadeia extrativista faz-se necessária para que políticas para a produção e conservação desta semente, intimamente ligada à conservação da espécie Araucária angustifólia, possam ser levadas a cabo. Assim, o objetivo deste trabalho é realizar uma análise preliminar sobre os aspectos produtivos e comerciais que envolvem o pinhão no estado do Paraná.

\section{MATERIAIS E MÉTODOS}

Os dados utilizados neste artigo foram coletados junto as seguintes fontes: Instituto Brasileiro de Geografia e Estatística (IBGE), Secretaria do Estado da Agricultura e Abastecimento do Paraná (SEAB-PR), Centros de Abastecimento (CEASA) e pontos de varejo e se referem a volumes comercializados, preços nos vários níveis de comercialização, preços por período e procedências e destinos do comércio. Além de dados quantitativos foram levantadas as características desta cadeia extrativista através de entrevistas com especialistas e pessoas envolvidas com a mesma.

O método utilizado para avaliar os ganhos nos diferentes níveis de comercialização foi o cálculo dos índices de Margem e Markup de Comercialização.

A Margem Bruta de Comercialização refere-se ao valor percentual de participação de cada um dos níveis de comercialização na formação do preço final do produto. A margem total $(\mathrm{Mt})$ refere-se à diferença do preço pago pelo consumidor e o preço pago ao produtor. Algebricamente Mt pode ser também calculada pelo somatório de todas as margens dos níveis de comercialização (Kotler, 2000). Na tabela 1 é apresentado o formulário para o cálculo da Margem Bruta de Comercialização.

Em termos absolutos o Markup (Mk) corresponde a margem de comercialização, a qual refere-se a diferença de preços em diferentes níveis do sistema comercial. Em termos relativos, o Markup mostra o percentual de aumento entre os preços de venda e de compra relativamente ao preço de compra, ou, entre o preço de venda e o custo de produção relativamente ao custo de produção (Kotler, 2000). As fórmulas de cálculo para Markup Total (Mkt), Intermediário (Mki), Atacado (Mka) e Varejo (Mkv) são apresentadas na tabela 2 . 
Tabela 1: Fórmulas para o cálculo do margem de comercialização

Table 1: Formulas used for calculations of marketing margins

\begin{tabular}{|c|c|c|}
\hline Margem de Comercialização & Valor Absoluto & Valor Relativo \\
\hline Total $(\mathrm{Mt})$ & $\mathrm{Pv}-\mathrm{Pp}$ & {$[(\mathrm{Pv}-\mathrm{Pp}) / \mathrm{Pv}]^{*} 100$} \\
\hline Intermediário $(\mathrm{Mi})$ & $\mathrm{Pi}-\mathrm{Pp}$ & {$[(\mathrm{Pi}-\mathrm{Pp}) / \mathrm{Pv}]^{*} 100$} \\
\hline Atacado $(\mathrm{Ma})$ & $\mathrm{Pa}-\mathrm{Pi}$ & {$[(\mathrm{Pa}-\mathrm{Pi}) / \mathrm{Pv}]^{*} 100$} \\
\hline Varejo $(\mathrm{Mv})$ & $\mathrm{Pv}-\mathrm{Pa}$ & {$[(\mathrm{Pv}-\mathrm{Pa}) / \mathrm{Pv}]^{*} 100$} \\
\hline
\end{tabular}

Onde:

$\mathrm{Pv}=$ preço pago pelo consumidor final no varejo;

$\mathrm{Pi}=$ preço de venda do intermediário para o atacadista;

$\mathrm{Pa}=$ preço de venda pelo atacadista;

$\mathrm{Pp}=$ preço pago ao proprietário

Tabela 2: Fórmulas para o cálculo do Markup de Comercialização

Table 2: $\quad$ Formulas used for markup calculations

\begin{tabular}{|c|c|c|}
\hline Markup & Valor Absoluto & Valor Relativo \\
\hline Total (Mkt) & $\mathrm{Pv}-\mathrm{Pp}$ & {$[(\mathrm{Pv}-\mathrm{Pp}) / \mathrm{Pp}]^{*} 100$} \\
\hline Intermediário (Mki) & $\mathrm{Pi}-\mathrm{Pp}$ & {$[(\mathrm{Pi}-\mathrm{Pp}) / \mathrm{Pp}]^{*} 100$} \\
\hline Atacado (Mka) & $\mathrm{Pa}-\mathrm{Pi}$ & {$[(\mathrm{Pa}-\mathrm{Pi}) / \mathrm{Pi}]^{*} 100$} \\
\hline Varejo (Mkv) & $\mathrm{Pv}-\mathrm{Pa}$ & {$[(\mathrm{Pv}-\mathrm{Pa}) / \mathrm{Pa}]^{*} 100$} \\
\hline
\end{tabular}

\section{RESULTADOS}

O fluxo de comercialização do pinhão caracteriza-se essencialmente pelo baixíssimo grau de industrialização, decorrente basicamente de aspectos culturais, restrições ditadas pela sazonalidade e quantidade produzida do produto. No que se refere aos fatores culturais, a semente é normalmente comprada já preparada, em feiras livres ou pontos do varejo especializados, ou in natura para ser preparada no domicílio do consumidor. Não raro pode-se encontrar receitas preparadas em feiras ou em alguns pontos do varejo, porém esta forma corresponde a uma parcela bastante pequena de toda a quantidade consumida do produto.

Uma outra característica importante é a sazonalidade da produção, que associada ao elevado grau de perecividade restringe a comercialização do produto principalmente aos meses de março a junho. Deve-se ressaltar todavia, que esta concentração da comercialização somente nos meses de produção advém possivelmente do baixo grau de industrialização do produto.

O fluxograma da cadeia extrativa do pinhão é apresentado na figura 1. Este fluxo inicia-se na propriedade, com a coleta da semente, podendo ter quatro destinos, a saber: centros de abastecimento ${ }^{4}$, intermediários ${ }^{5}$, varejo $^{6}$ e consumidor. A partir dos centros de abastecimento, a produção pode ser direcionada para os intermediários ou para a rede de varejo. Os intermediários podem vender $o$ produto para os centros de abastecimento, rede de varejo ou diretamente para o consumidor.

\section{Extração $^{7}$}

A coleta representa uma atividade das mais difíceis, podendo ser efetuada de duas maneiras: coleta no solo, quando os pinhões caem naturalmente com a maturação das pinhas (conjunto de sementes na forma globular), ou pela derrubada destas prematuramente.

A atividade de coleta apresenta alguns problemas que podem inviabilizá-la do ponto de vista comercial. Primeiramente, a maturação das pinhas dá-se em diferentes épocas (cada variedade tem maturação em períodos diferentes) tornando a coleta incerta. Além disso, a quantidade coletada por árvore é

\footnotetext{
${ }^{4}$ Centrais atacadistas para os quais parte da produção agrícola e pecuaria são carreadas.

Os intermédiários são considerados dentro do conceito de canais de marketing, o conjunto de organizações independentes envolvidas no processo de disponibilização de um produto para o consumo (KOTLER, 2000).

$6 \mathrm{O}$ varejo inclui todas as atividades relativas à venda de produtos ou serviços diretamente aos consumidores finais.

${ }^{7} \mathrm{O}$ pinhão comercializado provém principalmente de povoamentos naturais, coletados via extrativismo.
} 
geralmente pequena, obrigando o retorno à mesma árvore várias vezes e ao percurso de uma grande área para coletar quantidade satisfatória. Outro fator que contribui para a inviabilidade dessa técnica é a distribuição das árvores produtoras que ocorrem em baixa densidade e/ou são de difícil acesso.

A derrubada das pinhas minimiza alguns dos problemas encontrados na coleta, porém, existem outras dificuldades como o acesso aos galhos que contém as pinhas, transporte e escolha das árvores. A derrubada, geralmente é realizada com a utilização de uma vara de bambu, e pode ser feita à partir do chão ou subindo-se nos galhos do pinheiro e árvores circunvizinhas. $\mathrm{O}$ maior agravante de periculosidade nesse processo, é a subida nas árvores, pois geralmente é feita sem a utilização de equipamentos de segurança. No que concerne ao transporte, a desvantagem da derrubada é o volume extra que deve ser carregado. Uma alternativa é abrir as pinhas in loco (debulhar), o que nem sempre é possível, principalmente quando prematuras.

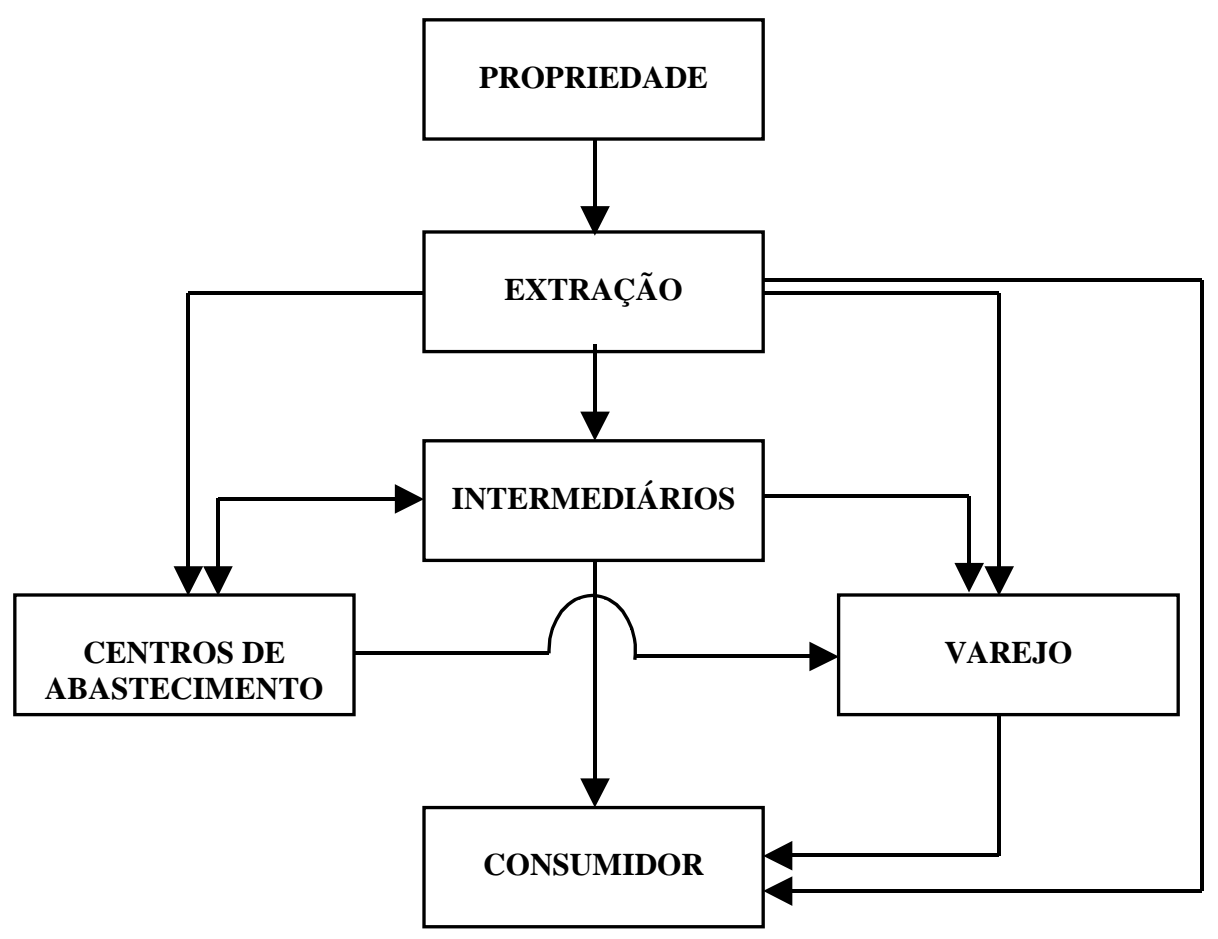

Figura 1: Fluxograma da Cadeia extrativa do Pinhão

Figure 1: Fluxogram for the Harvesting Pinhão Chain

Fonte: Pesquisa realizada pelos autores

\section{Proprietários e Intermediários}

Os intermediários desempenham um importante papel na cadeia extrativa do pinhão, principalmente em localidades onde a produção é pequena, inviabilizando o transporte pelos extratores para os centros de varejo, onde o produto alcança maiores preços. O preço de compra na propriedade é uma variável de difícil inferência, uma vez que, como observado no fluxograma de comercialização (fig. 1), o produto à partir da propriedade pode ter vários destinos. Para o dono desta, certamente a melhor alternativa é, quando possível, fazer a comercialização diretamente com o consumidor, ficando com toda a margem de comercialização. Porém nem sempre esta alternativa é viável, uma vez que na maioria dos casos os centros produtores estão localizados distante dos centros consumidores. Nesse ponto existem dois entraves para os pequenos proprietários: 1) a quantidade produzida não compensa ser transportada ou 2) os proprietários/coletores 
não dispõe de meios de transporte.

Neste caso, existe uma outra possibilidade, que é a entrada de um intermediário comprando de vários proprietários pequenos. O preço pago por um intermediário pode variar de $\mathrm{R} \$ 0,10$ a $\mathrm{R} \$ 0,50$ o quilograma. Os preços mais baixos são praticados quando $\mathrm{o}$ intermediário se responsabiliza pela extração do produto na propriedade. Os intermediários podem vender para os centros atacadistas a preços que podem variar de $R \$ 0,50$ a $R \$ 1,10$ o quilograma.

\section{Atacadistas}

O preço de compra pelos atacadistas (centros de abastecimento), varia de $\mathrm{R} \$ 0,50$ à $\mathrm{R} \$ 1,10$ por quilo sendo o mesmo repassado para o varejo a um preço médio de $\mathrm{R} \$ 1,03 / \mathrm{Kg}$ (CEASA/ Curitiba). Os atacadistas compram o produto de intermediários ou dos próprios produtores. Parte do volume que chega ao CEASA/Curitiba é proveniente do próprio estado do Paraná, e outra parte provém de outros estados produtores.

\section{Varejistas}

$\mathrm{O}$ varejista pode receber o produto a partir de três procedências:

- direto da propriedade/extrator;

- dos centros de abastecimento;

- de intermediários.

De modo geral, quando é possível negociar diretamente com os proprietários sempre há possibilidade de se aumentar a margem de comercialização do varejista. $\mathrm{Na}$ maioria das vezes esta não é uma prática viável, uma vez que existe um alto custo e tempo de transporte, em contraposição às baixas quantidades comercializadas. A alternativa nesse caso é comprar no atacado ou de intermediários. No estado do Paraná , o preço médio praticado pelo atacado (CEASA) é $\mathrm{R} \$ 1,03 / \mathrm{kg}$. O preço de venda no varejo varia de $\mathrm{R} \$ 1,00$ à $\mathrm{R} \$ 1,50 / \mathrm{Kg}$, com uma média de $\mathrm{R} \$ 1,25$ / $\mathrm{Kg}$. Este preço varia também em função da época do ano sendo que no começo e no final da época de produção, o pinhão chega a atingir preços mais elevados do que os mencionados, chegando em alguns casos a mais de 2,00 $\mathrm{R} \$ / \mathrm{kg}$ (tab. 3).

Tabela 3: Série temporal do preço médio (US\$) do pinhão na CEASA de Curitiba

Table 3: Time series of average price (US\$) of pinhão in CEASA of Curitiba

\begin{tabular}{|c|c|c|c|c|c|}
\hline Mês / Ano & 1996 & 1997 & 1998 & 1999 & 2000 \\
\hline Março & 1.25 & 0.85 & 0.57 & 0.39 & 0.43 \\
\hline Abril & 0.76 & 1.47 & 0.46 & 0.35 & 0.45 \\
\hline Maio & 0.85 & 0.67 & 0.38 & 0.47 & 0.46 \\
\hline Junho & 1.90 & 0.96 & 0.75 & 0.49 & 0.74 \\
\hline Julho & 2.49 & 0.76 & 0.47 & 0.43 & 0.92 \\
\hline
\end{tabular}

Fonte : CEASA/Curitiba

\section{Consumidores}

São todas as famílias ou pessoas individualmente, das mais variadas classes sociais, que compram o pinhão diretamente dos extratores, dos intermediários ou nos vários pontos do varejo para consumi-lo.

\section{Aspectos Produtivos e Comerciais}

Produção

Segundo a Secretaria de Estado da Agricultura e do Abastecimento - SEAB, na safra de 97/98 a produção total do estado foi de $1.587 .460 \mathrm{~kg}$, sendo que deste volume, os municípios de Campo Largo e General Carneiro foram mais expressivos, com respectivamente 7,5 e $6,3 \%$ figura 2 . 


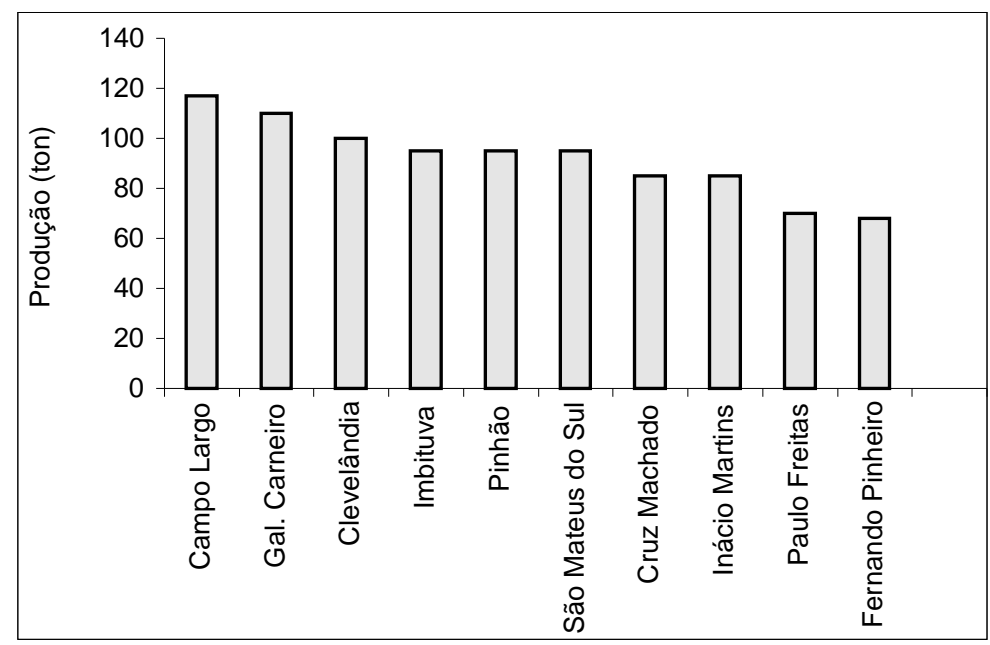

Figura 2: Principais municípios produtores de pinhão no Estado do Paraná safra 98/99

Figure 2: Main Counties producers of pinhão in the State of Parana - harvest 98/99

FONTE: Secretaria do Estado da Agricultura e do Abastecimento - 2000

De acordo com os dados do CEASA, contidos na Tabela 4, a quantidade de pinhão comercializada no Paraná em 2001 foi 952.300 $\mathrm{kg}$ a um preço médio de $\mathrm{R} \$ 1,03 / \mathrm{kg}$. Deste volume o Paraná participou $344.239 \mathrm{~kg}$, São Paulo com 308.600 kg, Minas Gerais com
$150.790 \mathrm{~kg}$ e os outros estados participaram com $148.691 \mathrm{~kg}$. Do volume comercializado proveniente do Paraná, o município de Pinhão foi responsável por $69,8 \%$, seguido por Guarapuava com $11,1 \%$.

Tabela 4: Preço médio de venda do pinhão no Paraná e procedência do volume comercializado durante o ano de 2001

Table 4: Average sales prices of Pinhão from Parana and volume sold during year of 2001.

\begin{tabular}{|c|c|c|}
\hline Procedência & Preço médio $(\mathrm{R} \$ / \mathrm{kg})$ & Volume \\
\hline Espírito Santo & 1,65 & 500 \\
\hline Minas Gerais & 0,98 & 150.790 \\
\hline Paraná & 1,03 & 344.239 \\
\hline Rio Grande do Sul & 1,40 & 116.851 \\
\hline Santa Catarina & 1,30 & 31.340 \\
\hline São Paulo & 0,86 & 308.600 \\
\hline Total & 1,03 & 952.320 \\
\hline
\end{tabular}

Fonte: Dados coletados junto à CEASA-PR e ao Deral da SEAB-PR

O preço do pinhão na propriedade é muito inferior ao preço praticado pelo mercado, atingindo, segundo alguns proprietários rurais, $\mathrm{R} \$ 0,10$ por quilograma vendido.

\section{Margem Bruta e Markup de Comercialização}

$\mathrm{Na}$ tabela 5 estão apresentadas as Margens Brutas de Comercialização no interior da cadeia extrativa. Observa-se que $40 \%$ desta margem é apropriada pelo intermediário enquanto o atacadista apropria-se em média $18,4 \%$, o varejista $17,6 \%$, restando $24 \%$ para o proprietário. A Margem Total de Comercialização é de $76 \%$. 
Tabela 5: Margem Bruta de Comercialização

Table 5: Gross marketing margins

\begin{tabular}{|c|c|c|c|c|c|c|c|c|}
\hline \multicolumn{4}{|c|}{ PREÇOS (R\$) } & \multicolumn{4}{|c|}{ MARGEM (\%) } & \multirow{2}{*}{$\begin{array}{c}\text { Participação } \\
\text { do } \\
\text { Proprietário }\end{array}$} \\
\hline $\begin{array}{c}\text { Proprietário } \\
\text { (Pp) }\end{array}$ & $\begin{array}{c}\text { Intermediário } \\
(\mathrm{Pi})\end{array}$ & $\begin{array}{l}\text { Atacado } \\
(\mathrm{Pa})\end{array}$ & $\begin{array}{l}\text { Consumidor } \\
(\mathrm{Pv})\end{array}$ & $\begin{array}{l}\text { Intermediário } \\
\text { (Mi) }\end{array}$ & $\begin{array}{c}\text { Atacado } \\
\text { (Ma) }\end{array}$ & $\begin{array}{l}\text { Varejo } \\
(\mathrm{Mv})\end{array}$ & $\begin{array}{l}\text { Total } \\
(\mathrm{Mt})\end{array}$ & \\
\hline 0,30 & $\mathrm{O}, 80$ & 1,03 & 1,25 & $40 \%$ & $18,4 \%$ & $17,6 \%$ & $76 \%$ & $24 \%$ \\
\hline
\end{tabular}

Fonte: Autores

A tabela 6 apresenta os preços médios e Markups de Comercialização. Observa-se que o intermediário recebe uma remuneração de $167 \%$ sobre o preço de compra enquanto o

Tabela 6: Markup de Comercialização

Table 6: $\quad$ Marketing markups

\begin{tabular}{|c|c|c|c|c|c|c|c|}
\hline \multicolumn{4}{|c|}{ PREÇOS (R\$) } & \multicolumn{4}{c|}{ MARKUP (\%) } \\
\hline $\begin{array}{c}\text { Proprietário } \\
(\mathrm{Pp})\end{array}$ & $\begin{array}{c}\text { Intermediário } \\
(\mathrm{Pi})\end{array}$ & $\begin{array}{c}\text { Atacado } \\
(\mathrm{Pa})\end{array}$ & $\begin{array}{c}\text { Varejo } \\
(\mathrm{Pv})\end{array}$ & $\begin{array}{c}\text { Intermediário } \\
(\mathrm{Mki})\end{array}$ & $\begin{array}{c}\text { Atacado } \\
(\mathrm{Mka})\end{array}$ & $\begin{array}{c}\text { Varejo } \\
(\mathrm{Mkv})\end{array}$ & $\begin{array}{c}\text { Total } \\
(\mathrm{Mkt})\end{array}$ \\
\hline 0,30 & $\mathrm{O}, 80$ & 1,03 & 1,25 & $167 \%$ & $29 \%$ & $21 \%$ & $317 \%$ \\
\hline
\end{tabular}

Fonte: Autores

\section{CONCLUSÕES E RECOMENDAÇÕES}

- A estrutura da cadeia extrativa do pinhão é bastante simplificada como ficou evidenciado pelo fluxograma apresentando suas principais ligações. Nele não se verifica nenhum processo industrial, a exemplo do que acontece com outras amêndoas. Possivelmente um dos obstáculos à comercialização de um volume maior deste produto, deve-se à indisponibilidade de técnicas mais aprimoradas para sua conservação e industrialização.

- As Margens e Markups de Comercialização mostram que a parte mais expressiva da lucratividade no interior desta cadeia extrativa é apropriada pelos intermediários. Estes são responsáveis por mais da metade do total de acréscimo de preço no produto para o consumidor final. Do ponto de vista do proprietário, observa-se que o baixo preço alcançado pelo produto neste nível de comercialização desistimula esta atividade.

- Os preços baixos significam de certo modo uma relativa abundância do produto para o nível de consumo atual nas regiões produtoras. Entretanto sabe-se que a árvore da qual é proveniente o pinhão merece cuidados para evitar sua extinção.

- Para que o consumo e a produção de pinhão se desenvolva, estendendo este hábito alimentar para outras regiões e países, faz-se atacadista recebe em média $29 \%$ e o varejista $21 \%$. Os preços praticados pelos proprietários sofrem um acréscimo de $317 \%$ até chegar ao consumidor final. necessário pesquisas sobre o desenvolvimento de técnicas de conservação e industrialização disponibilizando-o nas demais estações do ano.

\section{REFERÊNCIAS}

CEASA. Estatísticas do comércio de pinhão. Curitiba, 2000.

IAP: Coletânea SERFLOR: Sistema Estadual de Reposição Florestal. Curitiba, IAP Governo do Estado do Paraná, 2000.

IUFRO. Meeting on forestry problems of the genus Araucaria, vol. 1. Curitiba, 1979.

KOTLER,P. Administração de marketing: a edição do novo milênio. 10 ed. São Paulo: Prentice Hall, 2000.

RIZZINI, C. T. Árvores e madeiras úteis do Brasil: manual de dendrologia brasileira. São Paulo: Edgar Blücher, 1971.

SANQUETTA C. R. Pinheiro do Paraná: lendas e realidades. Curitiba: FUPEF, 1999.

SEAB. Estatísticas de produção e preços, Curitiba, 2000.

www.ibge.gov.br : IBGE: Página da internet para recuperação eletrônica de dados. 